\title{
A Chinese herbal decoction, Jian-Pi-Yi-Shen, regulates the expressions of erythropoietin and pro-inflammatory cytokines in cultured cells
}

Jianping Chen ${ }^{1}$, Amy G. W. Gong ${ }^{2}$, Xiaoyan Liu', Zhonggui Li ${ }^{1}$, Airong Qi ${ }^{1}$, Tina T. X. Dong ${ }^{2}$, Tiegang Yi ${ }^{*}$, Karl W. K. Tsim ${ }^{2}$ and Shunmin $\mathrm{Li}^{1,3^{*}}$

\begin{abstract}
Background: A Chinese herbal formula, namely Jian-Pi-Yi-Shen (JPYS), has been clinically prescribed for patients with chronic kidney disease associated-anemia, and which can improve the patient's immunological system. However, the mechanisms of JPYS involved in anemia and immune response have not been investigated. To study the role of JPYS in regulating hematopoietic and immunological functions, we investigated its activities on the expressions of erythropoietin and pro-inflammatory cytokines in cultured cells.

Methods: The standardized herbal extracts of JPYS $(0-30 \mu \mathrm{g} / \mathrm{ml})$ were applied onto cultured cells for $24-48 \mathrm{~h}$. Total RNA was collected from the treated cells and subjected to real-time quantitative PCR analysis. Cultured HEK293T cells, transfected with a construct composed of hypoxia response element tagged with a luciferase gene, i.e. pHRELuc, were treated with JPYS extracts $(1-30 \mu \mathrm{g} / \mathrm{ml})$ for $24 \mathrm{~h}$. The cell lysates were subjected to luciferase assay.

Results: The treatment with JPYS extract onto cultured HEK293T cells induced erythropoietin expression in a dosedependent manner, having the highest response by $\sim 50 \%$ of increase. In parallel, application of JPYS extract for $24 \mathrm{~h}$ stimulated expressions of interleukin (IL)-1 $\beta$, IL-6, and tumor necrosis factor (TNF)- $a$ in cultured RAW 264.7 macrophages. In contrast, the pretreatment with JPYS extract suppressed expressions of IL-1 $\beta, I L-6$, and TNF-a in lipopolysaccharide-induced macrophages.

Conclusions: These results confirmed the hematopoietic function of JPYS in regulating erythropoietin expression, as well as the bidirectional immune-modulatory roles of JPYS by regulating the expression of pro-inflammatory cytokines in cultures.
\end{abstract}

Keywords: Herbal medicine, Erythropoietin, Pro-inflammatory cytokines

\section{Background}

Jian-Pi-Yi-Shen (JPYS), a Chinese herbal decoction, is comprised of eight medicinal herbs, i.e., Astragali Radix, Atractylodis Macrocephalae Rhizoma, Dioscoreae Rhizoma, Cistanches Herba, Amomi Fructus Rotundus, Salviae Miltiorrhizae Radix et Rhizoma, Rhei Radix et Rhizoma, Glycyrrhizae Radix et Rhizoma Praeparata cum Melle. For over

\footnotetext{
*Correspondence: szyitiegang@126.com; zyylishunmin@163.com 'Shenzhen Key Laboratory of Hospital Chinese Medicine Preparation, Shenzhen Traditional Chinese Medicine Hospital, Guangzhou University of Chinese Medicine, Shenzhen, Guangdong, China

Full list of author information is available at the end of the article
}

20 years, JPYS has been clinically prescribed for patients with chronic kidney disease (CKD) associated-anemia, as it is believed to possess the efficacies of fortifying the spleen, tonifying the kidney, activating blood and resolving stasis. The most known cause of CKD anemia is an insufficient erythropoietin (EPO) production [1]. Recombinant human erythropoietin (rHuEPO) treatment has been employed to correct CKD associated-anemia as to improve the quality of patients' life. However, the potential side effects in cardiovascular system and weak response in some CKDassociated patients have restricted the usage of rHuEPO [2]. Apart from aforesaid treatments, a number of patients 
also may choose alternative therapeutic approaches, such as traditional Chinese medicine (TCM). Previous pharmacological studied also showed that JPYS could increase the hemoglobin level in the nephrectomised rats [3]. In parallel, clinical studies demonstrated that JPYS could regulate the level of interleukin (IL)-1, IL-2 and IL-6, as to improve patient's immunological system [4]. These studies indicated the trophic role of JPYS in blood nourishment and immunological system. However, the molecular mechanism of JPYS in nourishing blood and immune response remains unclear.

In recent findings, CKD anemia might result from a defective hypoxic signaling rather than the lack of EPOstimulating cells to synthesize EPO [5]. Bernhardt et al. (2010) reported that the hypoxia-inducible factor (HIF) signaling could increase EPO production in CKD patients [6]. We therefore speculated that JPYS might stimulate EPO expression via HIF signaling in achieving for the treatment of CKD anemia. Besides, the proinflammatory cytokines, produced by macrophages, play a vital role in the early stage of immune response [7]. Here, the transcriptional expression of EPO was analyzed in cultured kidney fibroblasts, HEK293T cells, and the regulatory role of JPYS in pro-inflammatory cytokine expression in RAW 264.7 macorphages was investigated.

\section{Methods}

\section{Chemical and reagent}

Echinacoside (Lot No. 111670-201,304), salvianolic acid B (Lot No. 110821-201,313), rhein (Lot No. 110757-200,206) were purchased from National Institutes for Food and Drug Control (Beijing, China). The purities of all marker chemicals were determined to be no less than $98 \%$ by normalization of peak areas, as revealed by HPLC-DAD. HPLC grade acetonitrile was purchased from Merck (Darmstadt, Germany), and ultrapure water was prepared using a Milli-Q purification system (Molsheim, France). Other reagents used here were of analytical grade. Reagents for cell cultures were purchased from Invitrogen Technologies (Carlsbad, CA). Mineral oil (hypoxia mimicry), lipopolysaccharide (LPS, a pro-inflammatory stimulus from Escherichia coli) and dexamethasone (an anti-inflammatory agent) were purchased from Sigma (St. Louis, MO) $[8,9]$.

\section{Plant materials and preparation of JPYS extract}

Plant materials: Astragali Radix (Lot. 150,621; roots of Astragalus membranaceus (Fisch.) Bge. var. mongholicus (Bge.) Hsiao), Atractylodis Macrocephalae Rhizoma (Lot. 141,230; rhizomes of Atractylodes macrocephala Koidz.), Dioscoreae Rhizoma (Lot. 150,615; rhizomes of Dioscorea opposite Thunb.), Cistanches Herba (Lot. 150,621; herbs of Cistanche deserticola Y.C. Ma), Amomi Fructus Rotundus (Lot. 150,617; fruits of Amomum kravanh
Pierre ex Gagnep.), Salviae Miltiorrhizae Radix et Rhizoma (Lot. 150,626; roots and rhizomes of Salvia miltiorrhiza Bge.), Rhei Radix et Rhizoma (Lot. 150,104; roots and rhizomes of Rheum palmatum L.), Glycyrrhizae Radix et Rhizoma Praeparata cum Melle (Lot. 150,615; roots and rhizomes of Glycyrrhiza uralensis Fisch.) were purchase from Shenzhen Huahui Pharmaceutical Co., Ltd. The plant materials were authenticated by Dr. Tina Dong based on their morphological characteristics. The voucher specimens were kept at Pharmaceutical Department, Shenzhen Traditional Chinese Medicine Hospital with number 2010015Z, 2010024ZZ, 2010037Z, 2040056Z, 202086Z, 2010006Z, 2010040Z and 2010008ZZ, respectively. Assurance of quality control for all the materials was validated according to the Chinese Pharmacopeia (China Pharmacopoeia Committee, 2015).

Astragali Radix (30 g), Atractylodis Macrocephalae Rhizoma (10 g), Dioscoreae Rhizoma (30 g), Cistanches Herba (10 g), Amomi Fructus Rotundus (10 g), Salviae Miltiorrhizae Radix et Rhizoma (15 g), Rhei Radix et Rhizoma (10 g), Glycyrrhizae Radix et Rhizoma Praeparata cum Melle (6 g) were weighed and extracted in boiling water $(1.2 \mathrm{~L})$ twice for $1 \mathrm{~h}$. After centrifugation $(13,000 \mathrm{rpm}, 10 \mathrm{~min})$, the supernatant was stored at $4{ }^{\circ} \mathrm{C}$ and filtered through a $0.22 \mu \mathrm{m}$ filter (Millipore Ireland Ltd., Ireland) before injection into HPLC system for analysis. For biological analysis, the extract was dried under reduced pressure to powder, and it was stored at $-80^{\circ}$ C. Before the treatment, the powder was re-dissolved with Milli-Q water and vortexed at room temperature to a concentration of $100 \mathrm{mg} / \mathrm{mL}$ of jujube extract. The solution was filtered through a $0.22 \mu \mathrm{m}$ filter before application onto cultured cells.

\section{Chromatographic conditions and instrumentation}

Validation HPLC method, as described in previous study [10], was performed on a Shimadzu (Kyoto, Japan) LC20AT system, which was equipped with a degasser, a binary pump, an autosampler and a diode array detector. The herbal extract was separated on Inertsil ODS-SP $(150 \mathrm{~mm} \times$ $4.6 \mathrm{~mm}, 5 \mu \mathrm{m}$ ) column. The mobile phase was composed of $0.1 \%$ formic acid (A) and acetonitrile (B) using the following gradient program: 0-5 min, $90 \% \mathrm{~A} ; 5-10 \mathrm{~min}$, 90\%-80\% A; $10-30$ min, $80 \%-60 \%$ A; $30-40$ min, $90 \%$ A; the flow rate was $1.0 \mathrm{ml} / \mathrm{min}$; the injection volume was $10 \mu \mathrm{L}$; the wavelength was $260 \mathrm{~nm}$. A pre-equilibration period of 10 min was used between each run. The content of tested markers was calculated using their calibration curve with regarding to the dilution factor and expressed as microgram per gram of dried extract weight.

\section{Cell culture and treatment}

Human embryonic kidney fibroblast (HEK) 293 T cells were obtained from American Type Culture Collection 
(ATCC, Manassas, VA). HEK293T T cells were cultured in Dulbecco's modified Eagle's medium (DMEM), supplemented with $10 \%$ fetal bovine serum, $100 \mu \mathrm{g} / \mathrm{mL}$ streptomycin, and $10 \%$ fetal bovine serum. Before plating, cells were rinsed with $5 \mathrm{~mL}$ phosphate buffered saline containing $1 \mathrm{mM}$ EDTA twice, and followed by using $1 \mathrm{~mL}$ trypsin/EDTA solution to detach the cells. For EPO mRNA analysis, cultured HEK293T cells $\left(5 \times 10^{4}\right.$ cells $/ \mathrm{ml})$ were treated with JPYS extracts $(1-30 \mu \mathrm{g} / \mathrm{mL})$ for $48 \mathrm{~h}$. For luciferase assay, cultured HEK293T cells, transfected with pHRE-Luc, were treated with JPYS extracts $(1-30 \mu \mathrm{g} / \mathrm{ml})$ for $24 \mathrm{~h}$. The mineral oil layering (oil: medium $=2: 1$ ) served as a positive control. The untreated culture was served as a blank control. RAW 264.7 murine macrophages from American Type Culture Collection (ATCC, Manassas, VA) were cultured in Dulbecco's modified Eagles medium (DMEM) supplemented with $100 \mathrm{IU} / \mathrm{mL}$ penicillin, $100 \mu \mathrm{g} / \mathrm{mL}$ streptomycin, and $10 \%$ heat in-active fetal bovine serum (FBS). Cells were incubated at $37{ }^{\circ} \mathrm{C}$ in a water-saturated $5 \% \mathrm{CO}_{2}$ incubator. Before plating, cells were dislodged from plate with a cell scraper (Corning Incorporated; Corning, NY). For cytokine upregulation, cultured RAW 264.7 macrophages were treated with JPYS extract at various concentrations $(0-30 \mu \mathrm{g} / \mathrm{ml})$ or LPS $(1 \mu \mathrm{g} / \mathrm{ml})$ for $24 \mathrm{~h}$. For cytokine downregulation, cultured RAW 264.7 macrophages were pre-treated with dexamethasone (Dex.; $10 \mu \mathrm{M}$; a positive control of suppressor) or JPYS extract at various concentrations $(0-30 \mu \mathrm{g} / \mathrm{ml})$ for $3 \mathrm{~h}$. The untreated culture was employed as a blank control. Then, LPS $(1.0 \mu \mathrm{g} / \mathrm{ml})$ was applied onto the cultures for $24 \mathrm{~h}$.

\section{Quantitative real-time PCR}

For the analyses of gene expressions in HEK293T cells and RAW 264.7 cells, the cultures were treated with JPYS extract. After the treatment, total RNA was isolated by RNAzol reagent (Molecular Research Center, Cincinnati, $\mathrm{OH}$ ), and reverse transcribed into cDNA according to the manufacturer's instructions (Invitrogen). Real-time PCR was performed by using FastStart Universal SYBR Green Master (ROX) according to the manufacturer's instructions (Roche Applied Science, Mannheim, Germany). The primers were: 5' - ACT TTC CGC AAA CTC TTC CG-3' (sense primer, S) and 5' - TGA ATG CTT CCT GCT CTG GG-3' (anti-sense primer, AS) for human EPO (330 bp; NM_000799.2); 5' - GCT TTA ACT TTG CTG GCC CCA GC-3' (S) and 5' - GCA GGG TCA GCA CTA CTT CGA AG-3' (AS) for human HIF-1 $\alpha$ (221 bp; NM_001530.3); 5' - AAATAC CTG TGG CCT TG-3' (S) and 5' - TTA GGA AGA CAC GGA TTC-3' (AS) for murine IL-1ß (296 bp; NM 008361); 5' - GGA GTA CCA TAG CTA CCT GG-3' (S) and 5' - CTA GGT TTG CCG AGT AGA TC-3' (AS) for murine IL-6 (283 bp; NM_031168); 5' - AGT GAC AAG CCT GTA GCC-3' (S) and 5'- AGG TTG ACT TTC TCC TGG-3' (AS) for murine TNF- $\alpha$ (251 bp; NM_013693). Glyceraldehyde 3 -phosphate dehydrogenase (GAPDH), a house-keeping gene, was used as an internal control in all cases, and its primer sequences were 5' - AAC GGA TTT GGC CGT ATT GG-3' (S) and 5' - CTT CCC GTT CAG CTC TGG G-3' (AS) (657 bp; NR_0215885). SYBR green signal was detected by ABI 7500 Fast Real-Time PCR system (Applied Biosystems, Foster City, CA). Transcript levels were quantified by using $\Delta \Delta \mathrm{Ct}$ value method [11], where the values of target genes were normalized by GAPDH in the same sample at first before comparison. PCR products were analyzed by gel electrophoresis and melting curve analysis, as to confirm the specific amplification.

\section{DNA transfection and luciferase assay}

Six hypoxia responsive elements (HRE: 5'-TCG AGG CCC TAC GTG CTG TCT CAC ACA GCC TGT CTG ACG-3') were synthesized, concatemerized and then cloned in tandem (head-to-tail orientation) into pBI-GL vectors (BD Biosciences Clontech, San Jose, CA) that has a downstream reporter of firefly luciferase gene: this vector was named as pHRE-Luc [12, 13]. Cultured HEK293T cells were transiently transfected with pHRELuc by the calcium phosphate precipitation method. The transfection efficient was over $80 \%$, as determined by another control plasmid of having a $\beta$-galactosidase, under a cytomergalovirus enhancer promoter. After $24 \mathrm{~h}$ of transfection, the luciferase assay was performed by a commercial kit (Tropix Inc., Bedford, MA). In brief, cultures were lysed by a buffer containing $100 \mathrm{mM}$ potassium phosphate buffer ( $\mathrm{pH}=7.8), 0.2 \%$ Triton X-100 and $1 \mathrm{mM}$ dithiothreitol. The luminescent reaction was quantified in a Tropix TR717TM Microplate Luminometer, and the activity was expressed as absorbance (up to $560 \mathrm{~nm}$ ) per mg of protein.

\section{Other assays}

Protein concentrations were measured by Bradford's method with a kit from Bio-Rad Laboratories (Hercules, CA). Individual data was expressed as Mean \pm standard deviation (SD). Statistical analyses were performed using one-way ANOVA (version 13.0, SPSS). Statistically significant changes were classified as significant $(*)$ where $p<0.05$, more significant (**) where $p<0.01$ and highly significant $(* * *)$ where $p<0.001$.

\section{Results}

\section{Preparation of standardized JPYS}

In order to chemically standardize JPYS extract, we employed the established HPLC fingerprint and quantification method to reveal its HPLC profile and quantify the main ingredients. A typical HPLC fingerprint 
at absorbance of $260 \mathrm{~nm}$ was developed for JPYS extract [10]: the fingerprint was served as an index for identification of JPYS. By using the respective individual standard, the chemical markers were identified from the extract, e.g. sodium danshensu, echinacoside, acteoside, calycosin 7-O- $\beta$-glucoside, salvianolic acid $B$, formononetin and rhein. Moreover, a rapid HPLCDAD method was developed to simultaneously quantify 3 main ingredients in JPYS extract. These

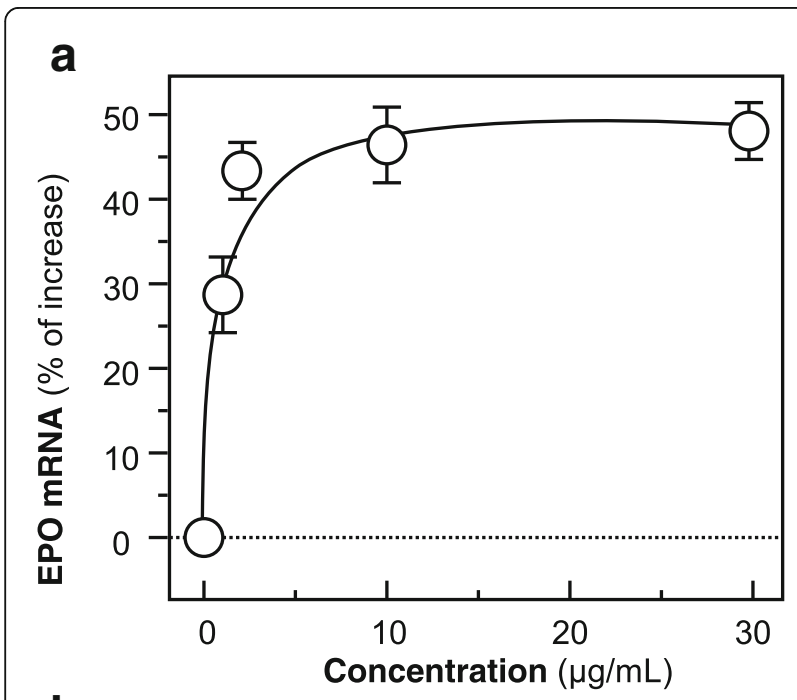

b

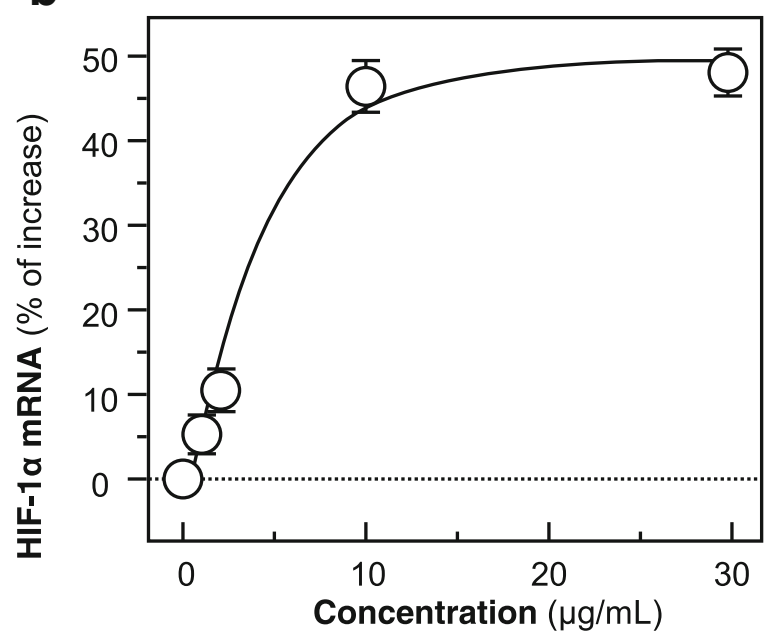

Fig. 1 JPYS stimulates the mRNA expressions of EPO and HIF-1a. a Cultured HEK293T cells $\left(5 \times 10^{4}\right.$ cells $\left./ \mathrm{ml}\right)$ were applied with JPYS extracts $(1-30 \mu \mathrm{g} / \mathrm{mL})$ for $48 \mathrm{~h}$. The level of EPO mRNA was detected by real-time PCR. b Cultured HEK293T cells were treated with JPYS extracts as in (a).The level of HIF-1a mRNA was detected by real-time PCR. GAPDH was used as an internal control for normalization. The mineral oil layering (oil: medium =2:1) served as a positive control, with the response about $80 \%$ and $150 \%$ of increase for expressions of EPO and HIF-1a mRNAs, respectively. Values are expressed as \% of increase to basal reading (untreated culture), and in Mean $\pm S D$, where $n=3$, each with triplicate samples. Statistical comparison was made with the control; ${ }^{*} p<0.05 ;{ }^{* *} p<0.01$ chemical markers included: echinacoside, salvianolic acid $B$ and rhein. A minimal requirement for the amounts of echinacoside, salvianolic acid B and rhein should be no less than $1.2 \mathrm{mg} / \mathrm{g}, 5.7 \mathrm{mg} / \mathrm{g}$ and $0.2 \mathrm{mg} /$ $\mathrm{g}$ of the dried extract. The yield of extraction was $32.59 \pm 1.1 \%(w / w$, Mean $\pm \mathrm{SD}, n=3)$. The extract being used here reached the aforesaid requirements, which guaranteed the repeatability of biological results.

\section{JPYS induces the expression of EPO}

To investigate the effect of JPYS, the extract of JPYS was applied onto cultured HEK293T cells for $48 \mathrm{~h}$. The concentrations chosen in this experiment did not show cytotoxicity on the cultures. Total RNA was collected from the treated cells and subjected to real-time quantitative PCR analysis by using specific primers flanking the EPO mRNA. The treatment with JPYS at various concentrations $(0-30 \mu \mathrm{g} / \mathrm{ml})$ induced the amount of EPO mRNA levels, and the effect of which was demonstrated to be dosedependent, having the highest response by $\sim 50 \%$ of

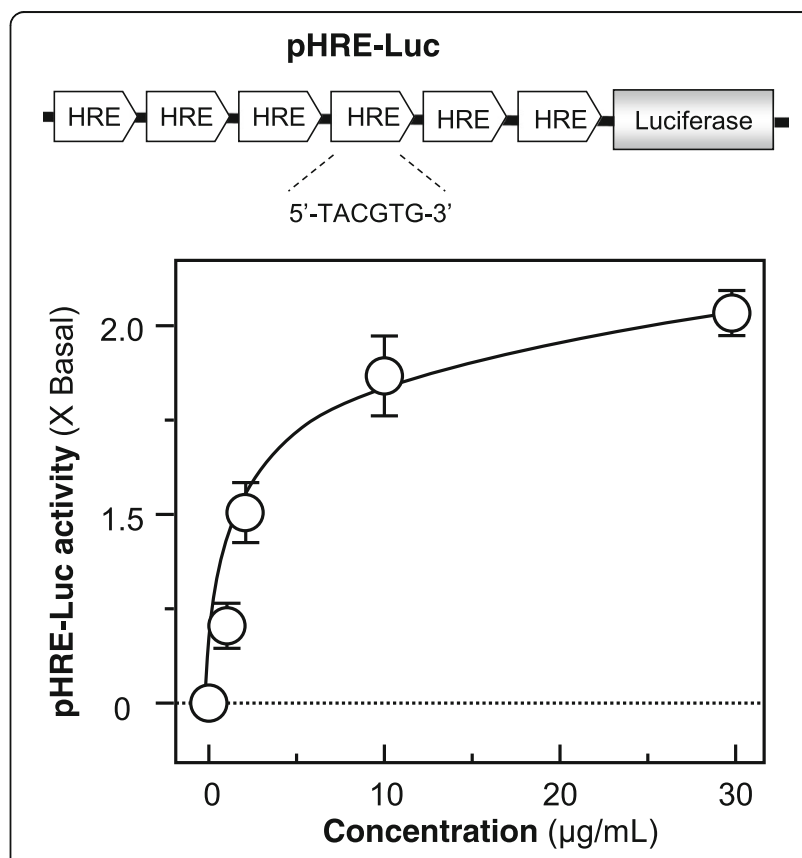

Fig. 2 JPYS induces HRE-mediated transcriptional activity. A luciferasereporter containing six HREs and a downstream luciferase-reporter gene, namely as pHRE-Luc, was used as a study tool (upper panel). Cultured HEK293T cells, transfected with pHRE-Luc, were treated with JPYS extracts $(1-30 \mu \mathrm{g} / \mathrm{ml})$ for $24 \mathrm{~h}$. The cell lysates were subjected to luciferase assay. The mineral oil layering (oil: medium $=2: 1$ ) served as a positive control, with the response about $70 \%$ of increase. Values are expressed as the fold of increase to basal reading (untreated culture), and they are in Mean $\pm S D$, where $n=3$, each with triplicate samples. Statistical comparison was made with the control; * $p<0.05 ;{ }^{* *} p<0.01$; *** $p<0.001$ 
increase (Fig. 1a). In order to reveal the regulatory mechanism of hypoxia inducible factor (HIF) in JPYS-induced HRE activation, the HIF signaling cascade involving in expression of HIF-1 $\alpha$ was analyzed. Here, the amount of mRNAs encoding HIF-1 $\alpha$ was determined in JPYS-treated HEK293T cells. The application of JPYS at various concentrations (0$30 \mu \mathrm{g} / \mathrm{ml}$ ) stimulated the amount of HIF-1 $\alpha$ mRNA level, and the effect was shown to be dosedependent, having the highest response by $\sim 50 \%$ of increase (Fig. 1b). The mineral oil layering, mimicking hypoxia condition, served as a positive control.

The activation of hypoxia-mediated signaling pathway is an inducer for EPO gene expression [14]. To reveal the transcriptional activity of HRE, a luciferase-reporter construct (pHRE-Luc), containing six HREs derived from the enhancer of EPO gene and tagged upstream of a luciferase gene was transfected into cultured HEK293T cells (Fig. 2, upper panel). To investigate the activation of HRE-mediated pathway, JPYS extracts at various concentrations were applied onto pHRE-Lucexpressed HEK293T cells. Here, JPYS extracts activated the HRE-mediated transcriptional activity in a dosedependent manner, having the highest response by $\sim 2$ fold of increase under treatment of $30 \mu \mathrm{g} / \mathrm{ml}$ (Fig. 2, lower panel). The mineral oil layering served as a positive control.

\section{JPYS regulates the expression of pro-inflammatory cytokines}

In order to reveal the immune-modulatory activities of JPYS at cellular level, macrophage activation involving induction of pro-inflammatory cytokines, e.g. IL-1 $\beta$, IL-6 and TNF- $\alpha$, were studied. JPYS extracts at various concentrations $(0-30 \mu \mathrm{g} / \mathrm{ml})$ were applied onto cultured RAW 264.7 cell for $24 \mathrm{~h}$. The extract induced the transcriptional expression of IL- $1 \beta$, IL- 6 and TNF- $\alpha$ in a concentrationdependent manner, having the highest response at $\sim 700$ fold, 15-fold and 20-fold under the treatment of JPYS extract at $30 \mu \mathrm{g} / \mathrm{ml}$ (Fig. 3). Furthermore, the effect of JPYS in suppressing the expression of pro-inflammatory cytokines under the LPS-challenged cell model. LPS $(1 \mu \mathrm{g} / \mathrm{ml})$ was applied onto the cultured macrophages, as to mimic the excessive activation. Dexamethasone served as a positive control. The pre-treatment with JPYS extract dosedependently suppressed the expressions of mRNAs encoding for IL-1 $\beta$, IL-6 and TNF- $\alpha$ in LPS-induced RAW 264.7 cells, having the highest reduction at $\sim 30 \%, \sim 35 \%$ and $50 \%$ under the pre-treatment of JPYS extract at $30 \mu \mathrm{g} / \mathrm{ml}$ (Fig. 4).
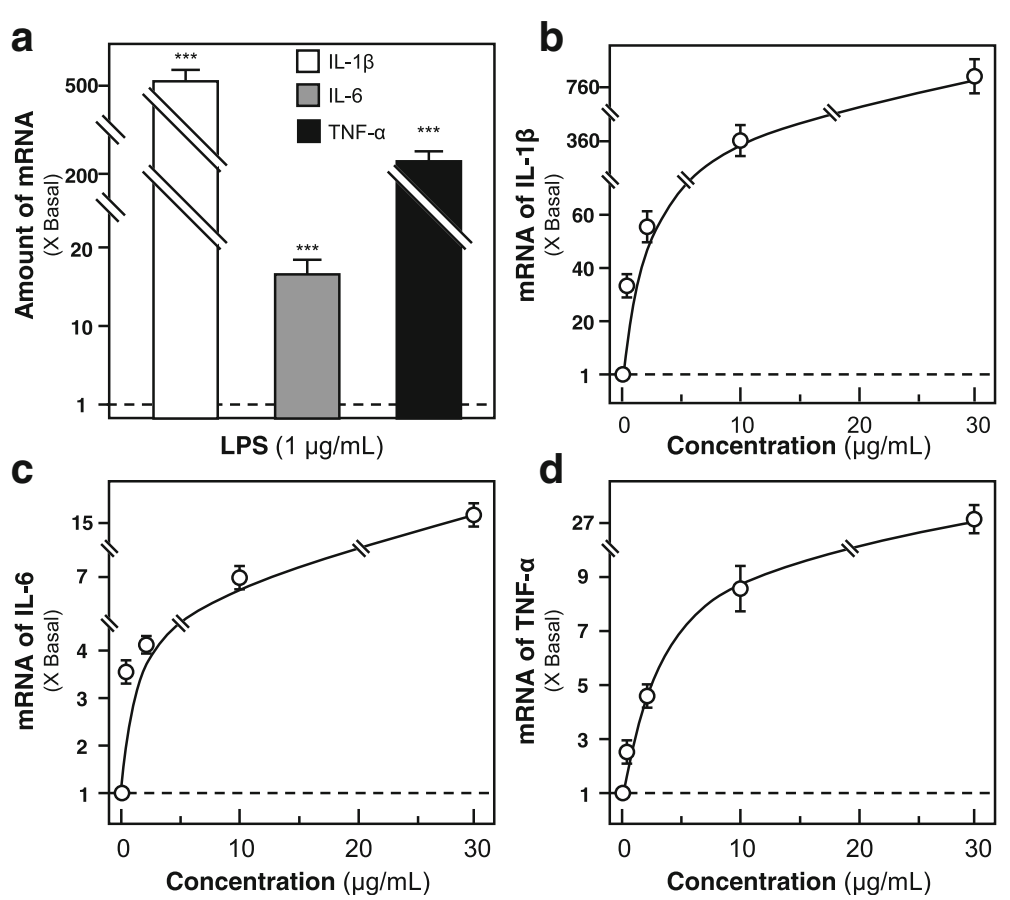

Fig. 3 JPYS induces the expressions of pro-inflammatory cytokines in cultured RAW 264.7 cells. a Cultured RAW 264.7 macrophages were treated with LPS $(1 \mathrm{\mu g} / \mathrm{ml})$ for $24 \mathrm{~h}$. LPS could robustly induce pro-inflammatory cytokine expression and which was employed as a positive control. b-d Cultured RAW 264.7 macrophages were treated with JPYS extract at various concentrations $(0-30 \mu \mathrm{g} / \mathrm{ml})$ for $24 \mathrm{~h}$. The mRNA levels of proinflammatory cytokines (IL-1 $\beta, \mathrm{LL}-6$ and TNF-a) were revealed by real time PCR. GAPDH mRNA served as an internal control for normalization. Data are expressed as the fold of increase to basal reading (untreated cultures), and in Mean \pm SD, where $n=3$, each with triplicate samples. Statistical comparison was made with the control; ${ }^{* *} p<0.001$ 
a

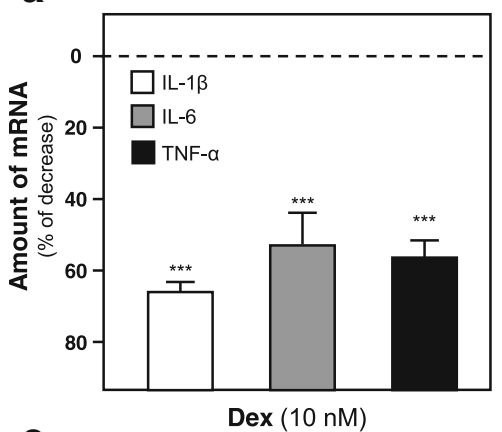

C

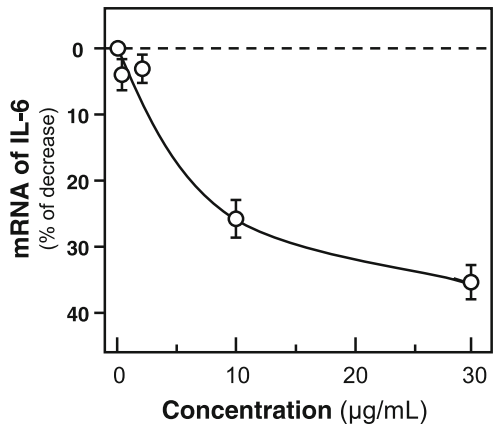

b

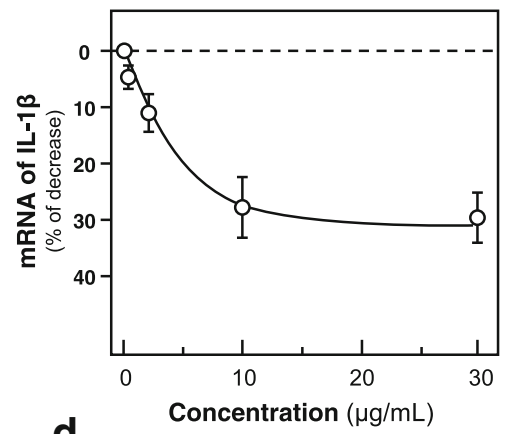

d

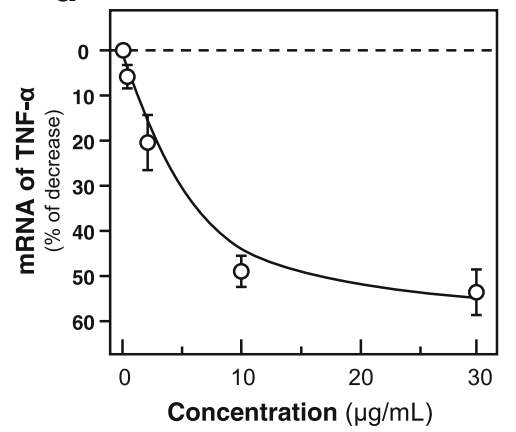

Fig. 4 JPYS suppresses the LPS-induced expression of pro-inflammatory cytokines in cultured macrophages. Cultured RAW 264.7 macrophages were pre-treated with Dexamethasone (Dex.; $10 \mu \mathrm{M}$; positive control of suppressor) (a) or JPYS extract (b-d) at various concentrations (0-30 $\mu \mathrm{g} /$ $\mathrm{ml}$ ) for $3 \mathrm{~h}$. Then, LPS $(1.0 \mathrm{\mu g} / \mathrm{ml})$ was applied onto the cultures for $24 \mathrm{~h}$ to mimic the chronic inflammation. The mRNA levels of proinflammatory cytokines IL-1 $\beta$, IL-6 and TNF-a were reveal by real time PCR. GAPDH was served as an internal control for normalization. Values are expressed as \% of the LPS-induced activation, and in Mean \pm SD, where $n=3$, each with triplicate samples. Statistical comparison was made with the control; * $p<0.05$; ${ }^{* *} p<0.01$; ${ }^{* *} p<0.001$

\section{Discussion}

CKD is a worldwide health issue affecting $\sim 7 \%$ of the population over the age of 30 [5]. Anemia is a very common major complication of CKD. EPO is a potent stimulator of erythropoiesis and produced mainly by kidney and liver: its production plays key role in the formation and production of red blood cell [15]. rHuEPO is commonly used to treat CKD associated-anemia. However, there are some side effects related to the usage of rHuEPO [2]. Traditional herbal medicines have been developed over thousands of years. Extensive experience and lots of clinical data for the therapy of CKD have been documented, which is very attractive for development of therapeutic drugs. Our current studies verified the hematopoietic function of JPYS by regulating EPO expression via hypoxia-inducible factor- $1 \alpha$ in kidney cells, suggesting that JPYS could be employed as an alternative medicine for the treatment of CKD associated-anemia. In support of this notion, our previous animal studies revealed that JPYS could increase the hemoglobin level [3].

Astragali Radix was served as sovereign medicine in JPYS formula. Astragali Radix is widely used as a health supplement to reinforce the body vital energy, and also well-known to improve the hypoxia condition. Regarding to EPO production, flavonoids have been reported to induce the expression of EPO [16]. Here, we assumed that flavonoids might be one of the active ingredients in JPYS that possessed the property to stimulate the EPO expression. In agreement with this notion, Zheng et al. (2011) reported that flavonoids from Astragali Radix including formononetin, ononin, calycosin, and calycosin-7-O- $\beta$ D-glucoside were able to stimulate EPO expression in cultured cells. And this effect was involved in the regulation of HIF signaling [16]. In our current study, seven compounds were identified in JPYS extract, which were reported to be relatively higher amount in the herbs and/ or possessed biological activities $[17,18]$. Thus, these parameters are recommended in making a standardized JPYS extract. Indeed, some chemical markers from Astragali Radix, such as formononetin and calycosin-7-O- $\beta$ D-glucoside, were also indentified in our current HPLC analysis. In addition, there are other flavonoids within other herbs in JPYS, such as echinacoside, acteoside and liquiritin. Whether these chemicals could induce EPO production however, which needs to be further studied.

On the other hand, immune deficiency is reported to complicate with loss of renal function and contributes significantly to patients with end-stage renal disease in 
terms of overall mortality and morbidity [19]. Macrophages are well-known to initiate specific defense mechanisms of our immune response. The pro-inflammatory cytokines, secreted by macrophages, play a vital role in early immune system $[7,9]$. IL-1 $\beta$, IL- 6 and TNF- $\alpha$ are the key pro-inflammatory cytokines playing critical roles in mediating the immune response [20]. Thus, these cytokines were being targeted in our current studies. The pro-inflammatory could activate macrophages, as the first line of defense against pathogens. Excessive activation of inflammatory cytokines, however, showed the damaging effects [9]. According to TCM theory, JPYS is able to fortify the spleen, which is referring to immune functions in modern medicine. Here, we found that JPYS exerted both pro- and anti-inflammatory activities in cultured RAW 264.7 cells. JPYS induced the expressions of pro-inflammatory cytokines, e.g. IL-1 $\beta$, IL-6 and TNF- $\alpha$. On the other hand, JPYS suppressed the expressions of LPS-induced cytokines. Hence, we proposed that JPYS extract possessed bi-directional immunemodulatory roles by regulating the expression of proinflammatory cytokines under different scenarios in macrophages. This notion is fully supported the clinical usage of JPYS for CKD patients having a complication of immune deficiency. In line with these findings, previous studies demonstrated that JPYS could regulate the level of IL-1, IL-2 and IL-6 in patients with kidney disease [4]. In addition, JPYS could improve patient's immune response by up regulating the level of $\mathrm{CD} 3+, \mathrm{CD} 4+, \mathrm{CD} 4$ +/CD8+ lymphocyte subgroups, as well as IL-2, and down regulating the level of CD8+, IL-1 and IL-6 [21].

\section{Conclusions}

In conclusion, JPYS induced the expression of EPO and activated the HRE-mediated transcriptional activity, which confirmed the hematopoietic function of JPYS. Moreover, JPYS possessed bi-directional immune-modulatory roles by regulating the expression of pro-inflammatory cytokines under different scenarios in macrophages, which may support the clinical usage of JYF for CKD patients having a complication of immune deficiency.

\section{Abbreviations}

CKD: chronic kidney disease; EPO: erythropoietin; HIF: hypoxia-inducible factor; HRE: hypoxia response element; IL: interleukin; JPYS: Jian-Pi-Yi-Shen; LPS: lipopolysaccharide; PCR: polymerase chain reaction; TCM: traditional Chinese medicine; TNF: tumor necrosis factor

\section{Acknowledgements}

Not applicable.

\section{Funding}

This work was supported by the Shenzhen Science and Technology Plan Project (JSGG20141017103353178, ZDSYS201606081515458,

JCYJ20150401163247213 and JCYJ20160428182041577), Ministry of Human Resources and Social Security of the People's Republic of China, Natural Science Foundation of Guangdong Province (2015A030310247), Traditional Chinese
Medicine Bureau of Guangdong Province (20151079, 20162124), and Health and Family Planning Commission of Shenzhen Municipality (201505015).

\section{Availability of data and materials}

The datasets used and/or analysed during the current study available from the corresponding author on reasonable request.

\section{Authors' contributions}

SL, TY and KT: Concept, design, literature search and manuscript review. XL and ZL: Lab work, analysis and interpretation of data. AQ and TD: Definition of intellectual content, manuscript review. JC and AG: Lab work, acquisition of data, drafting the manuscript. All authors have read and approved the manuscript.

Ethics approval and consent to participate

Not applicable.

\section{Consent for publication}

Not applicable.

\section{Competing interests}

The authors declare that they have no competing interests.

\section{Publisher's Note}

Springer Nature remains neutral with regard to jurisdictional claims in published maps and institutional affiliations.

\section{Author details}

${ }^{1}$ Shenzhen Key Laboratory of Hospital Chinese Medicine Preparation, Shenzhen Traditional Chinese Medicine Hospital, Guangzhou University of Chinese Medicine, Shenzhen, Guangdong, China. ${ }^{2}$ Division of Life Science and Center for Chinese Medicine, The Hong Kong University of Science and Technology, Hong Kong, China. ${ }^{3}$ Department of Nephrology, Shenzhen Traditional Chinese Medicine Hospital, Guangzhou University of Chinese Medicine, Shenzhen, Guangdong, China.

Received: 11 May 2017 Accepted: 27 February 2018

Published online: 03 April 2018

\section{References}

1. Cases-Amenos A, Martinez-Castelao A., Fort-Ros J, Bonal-Bastons J, Ruiz MP, Vallés-Prats M, Coll-Piera E, Galcerán-Gui JM, Investigadores del Estudio Micenas I. Prevalence of anaemia and its clinical management in patients with stages 3-5 chronic kidney disease not on dialysis in Catalonia: MICENAS I study. Nefrologia 2014; 34:189-198.

2. Palmer SC, Navaneethan SD, Craig JC, Johnson DW, Tonelli M, Garg AX, Pellegrini F, Ravani P, Jardine M, Perkovic V, Graziano G, McGee R, Nicolucci A, Tognoni G, Strippoli GF. Meta-analysis: erythropoiesis-stimulating agents in patients with chronic kidney disease. Ann Intern Med. 2010;153:23-33.

3. Li S, Zhou X, Zheng Y, Yi W. Effects of spleen-strengthening and kidneyinvigorating prescription on nutritional status in rats with chronic renal failure. J Guangzhou U Tradit. Chin Med. 2003;20:230-2.

4. Pan X, Yang D, Li S, Yi W, Jianpi Yishen FB. Formula regulates the level of IL-1, IL-2 and IL-6 in patient with uremia. J Guangming. Chin Med. 2008:23:1122-3

5. Garrido P, Ribeiro S, Fernandes J, Vala H, Rocha-Pereira P, Bronze-da-Rocha E, Belo L, Costa E, Santos-Silva A, Reis F. Resistance to recombinant human erythropoietin therapy in a rat model of chronic kidney disease associated anemia Int J Mol Sci. 2015:2015. https://doi.org/10.3390/ijms17010028.

6. Bernhardt WM, Wiesener MS, Scigalla P, Chou J, Schmieder RE, Günzler V, Eckardt KU. Inhibition of prolyl hydroxylases increases erythropoietin production in ESRD. J Am Soc Nephrol. 2010;21:2151-6.

7. Porcheray F, Viaud S, Rimaniol AC, Léone C, Samah B, Dereuddre-Bosquet N, Dormont D, Gras G. Macrophage activation switching: an asset for the resolution of inflammation. Clin Exp Immunol. 2005;142:481-9.

8. Chen J, Lam CT, Kong AY, Zhang WL, Zhan JY, Bi CW, Chan GK, Lam KY, Yao $P$, Dong TT, Tsim KW. The extract of Ziziphus jujuba fruit (jujube) induces expression of erythropoietin via hypoxia-inducible factor-1a in cultured Hep3B cells. Planta Med. 2014;80:1622-7.

9. Chen J, Du CY, Lam KY, Zhang WL, Lam CT, Yan AL, Yao P, Lau DT, Dong TT, Tsim KW. The standardized extract of Ziziphus jujuba fruit (jujube) regulates 
pro-inflammatory cytokine expression in cultured murine macrophages: suppression of lipopolysaccharide-stimulated NF-kB activity. Phytother Res. 2014;28:1527-32.

10. He T, Yu X, Zheng P, Zhou M, Zhang S, Chen J, Li S. Simultaneously content determination of echinacoside, salvianolic acid $B$ and rheic acid in Jianpi Yishen formula by HPLC. China Pharmaceuticals. 2017;26:25-7.

11. Winer J, Jung CKS, Shackel I, Williams PM. Development and validation of real-time quantitative reverse transcriptase-polymerase chain reaction for monitoring gene expression in cardiac myocytes in vitro. Anal Biochem. 1999;270:41-9

12. Post DE, Van Meir EG. Generation of bidirectional hypoxia/HIF-responsive expression vectors to target gene expression to hypoxic cells. Gene Ther. 2001;:1801-7.

13. Zheng KY, Choi RC, Xie HQ, Cheung AW, Guo AJ, Leung KW, Chen VP, Bi CW, Zhu KY, Chan GK, Fu Q, Lau DT, Dong TT, Zhao K, Tsim KW, 2010. The expression of erythropoietin triggered by Danggui Buxue tang, a Chinese herbal decoction prepared from radix Astragali and radix Angelicae Sinensis, is mediated by the hypoxia-inducible factor in cultured HEK293T cells. J Ethnopharmacol 2010;132:259-267.

14. Jelkmann $W, 2001$. The role of the liver in the production of thrombopoietin compared with erythropoietin. Eur J Gastroenterol Hepatol. 2001:13:791-801.

15. Ascensao JL, Bilgrami S, Zanjani ED, 2005. Erythropoietin: biology and clinical applications Am J Pediatr Hematol Oncol 2005;13:376-387.

16. Zheng KY, Choi RC, Cheung AW, Guo AJ, Bi CW, Zhu KY, Fu Q, Du Y, Zhang WL, Zhan JY, Duan R, Lau DT, Dong TT, Tsim KW. Flavonoids from radix Astragali induce the expression of erythropoietin in cultured cells: a signaling mediated via the accumulation of hypoxia-inducible factor-1 alpha. J Agric Food Chem. 2011;59:1697-704

17. Cui Q, Pan Y, Bai X, Zhang W, Chen L, Liu X. Systematic characterization of the metabolites of echinacoside and acteoside from Cistanche tubulosa in rat plasma, bile, urine and feces based on UPLC-ESI-Q-TOF-MS. Biomed Chromatogr. 2016;30:1406-15.

18. Liu D, Liu S, Yu X, Song N, Xu X, Hu J, Zhang T, Ding X. Salvianolic acid B prevents iodinated contrast media-induced acute renal injury in rats via the PI3K/Akt/Nrf2 pathway. Oxidative Med Cell Longev. 2016;2016:e7079487.

19. Betjes MG, Meijers RW, Litjens NH. Loss of renal function causes premature aging of the immune system. Blood Purif. 2013;36:173-8.

20. Du CY, Choi RC, Zheng KY, Dong TT, Lau DT, Tsim KW. Yu ping Feng san, an ancient Chinese herbal decoction containing Astragali radix, Atractylodis Macrocephalae Rhizoma and Saposhnikoviae radix, regulates the release of cytokines in murine macrophages. PLoS One. 2013;8:e78622.

21. Yang $D$, Lin J, Pan $X, Y i$ W, Li S. Effect of Jianpi Yishen formula on immune response in hemodialysis patient. J Neimenggu Tradit Chin Med. 2009:28:5-6.

\section{Submit your next manuscript to BioMed Central and we will help you at every step:}

- We accept pre-submission inquiries

- Our selector tool helps you to find the most relevant journal

- We provide round the clock customer support

- Convenient online submission

- Thorough peer review

- Inclusion in PubMed and all major indexing services

- Maximum visibility for your research

Submit your manuscript at www.biomedcentral.com/submit

) Biomed Central 